\title{
Amostragem por Importância para Estimar Valores Esperados: uma Abordagem com Heurísticas para Problemas Intervalares NP-Difíceis
}

A.B. LORETO ${ }^{1}$, Departamento de Matemática, UNISC, 96815-900 Santa Cruz do Sul, RS, Brasil

R. SILVA, L.V. TOSCANI, L. RIBEIRO ${ }^{2}$, Instituto de Informática, UFRGS, 91501-970 Porto Alegre, RS, Brasil.

D.M. CLAUDIO, L.A.S. LEAL ${ }^{3}$, Faculdade de Matemática, PUCRS, 90619900 Porto Alegre, RS, Brasil.

\begin{abstract}
Resumo. O presente trabalho tem como objetivo mostrar a possibilidade de aproximar um problema NP-Difícil da computação intervalar, através do uso de heurísticas com ênfase em algoritmos baseados no Método de Monte Carlo. A fim de apresentar heurísticas para o problema genérico de estimar numericamente, dada uma precisão $\varepsilon$, o valor esperado de uma função racional $f\left(x_{1}, \ldots, x_{n}\right)$ com $\left(x_{1}, \ldots, x_{n}\right) \in \prod_{k=1}^{n} \times I_{k}$ escolhidos de acordo com uma função de densidade de probabilidade $P\left(x_{1}, \ldots, x_{n}\right)$ conjunta, com $I_{k}$ sendo intervalos, mostramos para o particular caso de um grafo regular de grau 2, cujos vértices podem assumir somente dois valores -1 e +1 , o que restringe os intervalos a serem todos discretos $I_{k}=[-1,1] \cap \mathbb{Z}^{*}, k=1, \ldots, n$. Verificamos que o problema do valor esperado entre os pares de vértices adjacentes, tivera seu custo reduzido de $O\left(2^{n}\right)$ operações, através do cálculo exato, para $O\left(N_{\text {sample }}\right)$ que seria a complexidade encontrada quando aproximamos o resultado obtido pela Heurística, onde $N_{\text {sample é o número }}$ de amostras de configurações escolhidas através do processo de amostragem por importância no contexto do algoritmo de Metrópolis.
\end{abstract}

\section{Introdução}

Considerando que um vasto número dos problemas da computação intervalar é NP-Difícil, pode-se chegar a uma conclusão negativa: não se espera um algoritmo eficiente que resolva todos os problemas com tempo de processamento praticável [11]. Por outro lado, se considerarmos que as soluções destes problemas possam ser aproximadas, então existe uma grande possibilidade de conseguir resolvê-los em tempo aceitável. Neste caso torna-se possível aplicar heurísticas que são, em

\footnotetext{
${ }^{1}$ loreto@inf.ufrgs.br;

22rdasilva@inf.ufrgs.br, laira@inf.ufrgs.br, leila@inf.ufrgs.br;

3 dalcidio@inf.pucrs.br, liara@pucrs.br
} 
geral, procedimentos mais simples e flexíveis que os métodos exatos de solução, permitindo abordar problemas mais complexos, como os da classe NP-Difícil [1][2]. Contudo, é interessante observar que para cada problema que se deseja resolver, deve-se pesquisar uma heurística que melhor se adapte às condições do mesmo.

Nas pesquisas realizadas sobre computação intervalar no contexto da Estatística, verifica-se que problemas como variância, covariância e coeficiente de correlação com entradas intervalares são NP-difíceis quando computados pelo método da imagem intervalar [4]. Isto por si só, já é perturbador pois trata-se de problemas simples no contexto da estatística descritiva e deve ser salientado, até onde se sabe, que para estes problemas não foram testadas heurísticas capazes de solucioná-los ainda de maneira aproximada eficientemente com relação ao tamanho das entradas.

Um problema ainda mais complexo, da computação intervalar aplicado à teoria de probabilidades e que é o foco principal deste trabalho, seria calcular o valor esperado de uma função racional $f\left(x_{1}, \ldots, x_{n}\right)$, com $\left(x_{1}, \ldots, x_{n}\right) \in \prod_{k=1}^{n} \times I_{k} \subset \mathbb{R}^{n}$ com respeito a uma função de densidade de probabilidade $P\left(x_{1}, \ldots, x_{n}\right)$ conjunta, isto é, $P: \prod_{k=1}^{n} \times I_{k} \rightarrow \mathbb{R}^{n}$, onde $I_{k}, k=1, \ldots, n$ são intervalos. Conjectura-se que o problema na sua forma completa, ou seja, considerando correlação entre as variáveis, ou ainda que $P\left(x_{1}, \ldots, x_{n}\right)$ não é separável nas variáveis, é NP-Difícil. Nestas condições, nossa proposta para este trabalho seria intuir bem como heurísticas com ênfase em algoritmos baseados no Método Monte Carlo, podem aproximar soluções neste contexto. É importante ressaltar que a conjectura da NP-dificuldade do problema supra citado, está relacionado a NP-dificuldade de se calcular a imagem de uma função de $n$ variáveis reais com $n$ entradas intervalares (considerado como um dos problemas básicos da computação intervalar [3]).

O método Monte Carlo, na sua concepção, utiliza a geração de variáveis pseudoaleatórias para resolução de problemas [7]. Sua escolha deve-se ao fato de ser considerado muito simples e flexível para ser aplicado em problemas que envolvam muitos componentes e médias configuracionais, sob um grande número de estados.

Estimar o valor esperado (também chamado de esperança matemática) de uma função polinomial com este tipo de Heurística significa trocar valores médios, mais difíceis de serem calculados, por médias aritméticas simples em um processo conhecido como amostragem por importância.

O trabalho apresenta a seguinte estrutura: na seção 2 apresenta-se uma breve explicação sobre a complexidade computacional; na seção 3 descreve-se sobre o problema básico da computação intervalar, estado da arte e justificativa da NPdificuldade do problema (seção 3.1); na seção 4 apresenta-se o problema de estimar o valor esperado de uma função de várias variáveis e resultados provenientes da análise da complexidade sobre este problema; na seção 5 encontra-se um exemplo de como se aproximar eficientemente problemas de tempo exponencial através da aplicação da heurística conhecida por amostragem por importância (método Monte Carlo). Para isto, mostramos uma aplicação utilizando o algoritmo de Metrópolis para o cálculo de médias configuracionais em um grafo regular simples de grau 2; a seção 6 apresenta as conclusões do trabalho. 


\section{Um Pouco sobre Complexidade Computacional}

O termo complexidade refere-se, em geral, aos requerimentos de recursos necessários para que um algoritmo possa resolver um problema sob o ponto de vista computacional, ou seja, à quantidade de trabalho despendido pelo algoritmo. Para medir esta quantidade de trabalho é escolhida uma ou mais operações fundamentais e então são contados os números de execuções desta operação fundamental na execução do algoritmo [10]. A escolha de uma operação como operação fundamental é aceitável se o número de operações executadas pelo algoritmo é proporcional ao número de execuções desta operação [10].

A complexidade também pode ser vista como uma propriedade do problema, o que significa dar uma medida independente do tratamento dado ao problema, independente do caminho percorrido na busca da solução, portanto independente de algoritmos. Alguns problemas são bem comportados, isto é, permitem chegar a limites de complexidades bem definidos, outros estão em classes com contornos não bem claros [10].

Quanto à complexidade do problema de decisão, são definidas várias classes, como: P, NP, NP-Completo e NP-Difícil, entre outras. Os problemas da classe P certamente são tratáveis pois são resolvidos eficientemente por algoritmos determinísticos. Contudo, existem problemas cuja tratabilidade não está definida, que são os problemas da classe NP-Completo, de forma que sabemos que $\mathrm{P} \subset \mathrm{NP}$, mas não sabemos se $\mathrm{P} \neq \mathrm{NP}$, o que certamente acarretaria NP-Completo $\neq \varnothing$.

Um problema é dito intratável se a sua menor complexidade possível não é polinomial [10]. Para um problema pertencer a classe NP significa que existe pelo menos um algoritmo não-determinístico que o resolva em tempo polinomial. Um problema (não necessariamente da classe NP) é chamado NP-Difícil se todo o problema da classe NP pode ser reduzido a este. Se um problema da classe NP é NP-Difícil, este é chamado de NP-Completo. Para problemas das classes NP-Completo e NP-Difícil não se espera encontrar um algoritmo eficiente para verificá-lo [2], [3].

Identificar a tratabilidade e a intratabilidade dos problemas, mesmo aqueles que possuem algoritmos imediatos, é de extrema importância para os projetistas de algoritmos. Conhecendo as classes de complexidade a que os problemas pertencem, os projetistas poderiam ter uma medida real quanto às soluções disponíveis e a expectativa de melhorar esses resultados.

\section{Cálculo de Funções de Várias Variáveis Inter- valares}

Em [3] comenta-se sobre o problema conhecido entre a comunidadade da computação intervalar, que é de calcular a imagem de uma função $f\left(x_{1}, \ldots, x_{n}\right)$ de $n$ variáveis reais, com $n$ entradas intervalares $I_{i}=\left[\underline{x}_{i}, \bar{x}_{i}\right]$ e coeficientes racionais, ou seja, calcular $y=[\underline{y}, \bar{y}]=\left\{f\left(x_{1}, \ldots, x_{n}\right) \mid x_{1} \in I_{1}, \ldots, x_{n} \in I_{n}\right\}$.

Quando lidamos com quantidades físicas, geralmente os valores aproximados de

$\tilde{x}_{i}$, de uma quantidade física $x_{i}$ são conhecidos. Em alguns casos, são conhecidas as distribuições de probabilidades $\operatorname{dos}$ erros $\Delta x_{i}=\tilde{x}_{i}-x_{i}$ das diferentes medidas, 
porém em muitas outras situações práticas, é conhecido somente a exatidão $\Delta_{i}$ de cada medição. Em tais situações, tem-se somente informações sobre o valor real (desconhecido) $x_{i}$ na forma $\left|x_{i}-\widetilde{x}_{i}\right| \leq \Delta_{i} \mid$, ou seja, que o valor $x_{i}$ pertence ao intervalo $I_{i}=\left[\tilde{x}_{i}-\Delta_{i}, \tilde{x}_{i}+\Delta_{i}\right]$.

Usando uma relação conhecida entre $y$ e os valores de $x_{i}$ 's $\left(y=f\left(x_{1}, \ldots, x_{n}\right)\right)$, transforma-se os valores $x_{i}$ no valor da quantidade desejada $y$. Como resultado, se deseja conhecer o conjunto de possíveis valores de $y$. Para uma função contínua $f\left(x_{1}, \ldots, x_{n}\right)$, este conjunto é um intervalo denotado por $y=[y, \bar{y}]$.

Considerando que os dados de entrada $\tilde{x}_{i}$ não são precisos, o resultado $\tilde{y}$ do processamento de dados também não é preciso: este pode diferir do valor real (desconhecido) y da quantidade estimada. Entretanto, é necessário que o algoritmo, ou o processamento de dados, não retorne somente como uma estimativa numérica $\tilde{y}$, também o limite $\Delta$ de uma possível não exatidão desta estimativa. Dessa forma é possível deduzir o intervalo $y=[\tilde{y}-\Delta, \tilde{y}+\Delta]$ com os valores possíveis da quantidade desejada $y$. Contudo, necessita-se de métodos de processamento de dados que produzam não apenas resultados aproximados, mas com verificação automática de resultados. Neste caso utiliza-se a computação intervalar, ou seja, algoritmos de processamento intervalar.

Dos diversos problemas existentes na computação intervalar, o principal problema é a existência de métodos que freqüentemente retornam um intervalo delimitado $Y$ que superestima a imagem procurada $y: y \subseteq Y$ e $y \neq Y$.

Em decorrência deste fato pesquisadores estão tentando desenvolver algoritmos que permitem a menor superestimação possível, ou seja, o intervalo delimitado ótimo que coincide com a imagem desejada.

Dentro deste contexto, surge uma questão, muito importante: Pode-se calcular os extremos $y$ e $\bar{y}$ do intervalo $y$ em tempo razoável? Kreinovich [3] procurou responder esta questão analisando a complexidade do problema básico da computação intervalar. Verificou que o problema pertence à classe de problemas NP-Difícil, ou seja, é pelo menos tão difícil de resolver quanto qualquer problema NP.

\subsection{A NP-dificuldade do Problema}

É importante observar que a NP-dificuldade do problema básico da computação intervalar está relacionada com o processamento dos dados de entrada do problema. Neste problema, os dados de entrada de uma função contínua de várias variáveis $f\left(x_{1}, \ldots, x_{n}\right)$ são intervalos, ou seja $x_{1} \in I_{1}, \ldots, x_{n} \in I_{n}$, e em conseqüência, podese calcular a imagem desta função através de técnicas de métodos da computação intervalar. A seguir, citamos alguns dos métodos existentes:

- Extensão Intervalar: cada ocorrência da variável real $x$ de uma função real $f$ é substituída pela variável intervalar $I$ e cada operação real $\left(+,-,{ }^{*}, /\right)$ é substituída pela respectiva operação intervalar de tal modo que, quando $I=[x, x]$ então $F(I)=f(x)[6]$. Neste caso, geralmente encontra-se um intervalo delimitado $Y$ que superestima a imagem procurada $y$.

- Forma Centrada: a imagem de uma função $f\left(I_{1}, \ldots, I_{n}\right)$ é estimada como $f\left(I_{1}, \ldots, I_{n}\right) \subseteq f\left(\widetilde{x}_{1}, \ldots, \widetilde{x}_{n}\right)+\sum_{i=1}^{n} \frac{\partial f}{\partial x_{i}}\left(I_{1}, \ldots, I_{n}\right) \cdot\left[-\Delta_{i}, \Delta_{i}\right]$, onde $\widetilde{x}_{i}=\left(\underline{x}_{i}+\bar{x}_{i}\right) / 2$ é o ponto médio do intervalo e $\Delta_{i}=\left(\underline{x}_{i}-\bar{x}_{i}\right) / 2$ é o raio. Quando todos os inter- 
valos são iguais, a forma centrada não fornece a imagem desejada, pois retorna um intervalo centrado no ponto $f\left(\widetilde{x}_{1}, \ldots, \widetilde{x}_{n}\right)$. Como resultado da aplicação da forma centrada, obtém-se um intervalo superestimado e centrado em 0 (zero), isto é, um intervalo cujo extremo inferior é negativo [9].

- Imagem Intervalar: a imagem de uma função real $f$ em $I$ é o intervalo definido por $I m=I(f, I)=[\min f(x)|x \in I, \max f(x)| x \in I]$. Neste caso deve-se calcular os valores dos extremos inferior e superior do intervalo $I m$, isto é, no pior caso, tem-se que considerar todos os $n$ valores compreendidos entre $\underline{x}$ e $\bar{x}$ do intervalo $I$ para encontrar estes extremos. Como no problema de computar a imagem de uma função de várias variáveis $f\left(x_{1}, \ldots, x_{n}\right)$, tem-se $I_{i}$ intervalos, $i=1 . . m$, calcula-se o extremo inferior $y$ avaliando todos os valores de todos os $m$ intervalos (da mesma forma para $\bar{y}$ ), totalizando $m^{n}$ avaliações numéricas (operações fundamentais). Conforme o tamanho da entrada do problema, o tempo de processamento da computação cresce exponencialmente, e pela dificuldade do processamento de todos os dados, não se tem algoritmo de tempo de processamento polinomial conhecido.

Outro fato importante, e o que caracteriza o problema ser NP-Difícil, é que o problema em questão é um problema de decisão, pois deseja-se saber se existe o intervalo ótimo $y=[y, \bar{y}]$ que contenha a solução estimada ou aproximada do problema. A estimação na computação intervalar não fornece a imagem exata, e sim retorna um intervalo que contém o valor da imagem desejada. Esta imagem é chamada de estimativa do intervalo ótimo [11].

Na próxima seção descreveremos sobre a complexidade do problema de calcular o valor esperado de funções de $n$ variáveis, cujas variáveis estão definidas em intervalos.

\section{Cálculo do Valor Esperado de Funções de Várias Variáveis Intervalares}

O valor esperado de uma função de várias variáveis $f\left(x_{1}, \ldots, x_{n}\right) \in \mathbb{R}^{n}$, com $x_{1} \in$ $I_{1}, \ldots, x_{n} \in I_{n}$ é definida por

$$
E\left(f\left(x_{1}, \ldots, x_{n}\right)\right)=\int_{x_{1} \in I_{1}} \ldots \int_{x_{n} \in I_{n}} f\left(x_{1}, \ldots, x_{n}\right) P\left(x_{1}, \ldots, x_{n}\right) d x_{1} \ldots d x_{n},
$$

onde $P\left(x_{1}, \ldots, x_{n}\right)$ é a função densidade de probabilidade conjunta, tal que

$$
\int_{x_{1} \in I_{1}} \ldots \int_{x_{n} \in I_{n}} P\left(x_{1}, \ldots, x_{n}\right) d x_{1} \ldots d x_{n}=1 .
$$

Neste contexto, assume-se que as variáveis aleatórias $x_{1}, \ldots, x_{n}$ podem ser correlacionadas ou independentes. Ao considerar o problema correlacionado, o mesmo torna-se análogo ao problema de computar valores de funções racionais com entradas intervalares, ou seja, computar a imagem de uma função racional $f\left(x_{1}, \ldots, x_{n}\right)$ de $n$ variáveis reais, $n$ entradas intervalares $I_{k}=\left[\underline{x}_{k}, \bar{x}_{k}\right]$, com coeficientes racionais. 
Em [3], Kreinovich et al provaram que o problema de computar $y=[y, \bar{y}]=$ $f\left(I_{1}, \ldots, I_{n}\right)=\left\{y \mid y=f\left(\left\{x_{k}\right\}_{k=1}^{n}\right), x_{k} \in I_{k}\right\}$ é NP-Difícil mesmo para funções polinomiais, com uma certa flexibilidade ou exatidão $\varepsilon>0$ para os extremos $y$ e $\bar{y}$, isto é, os números racionais $|\widetilde{y}-y| \leq \varepsilon$ e $|\overline{\widetilde{y}}-y| \leq \varepsilon$.

Convém salientar que o problema de estimar o valor esperado da uma função de várias variáveis $f\left(x_{1}, \ldots, x_{n}\right) \in \mathbb{R}^{n}$, com $x_{1} \in I_{1}, \ldots, x_{n} \in I_{n}$ não havia sido abordado por pesquisadores da área de computação intervalar. Em conseqüência, não é possível realizar comparações com a utilização de outras heurísticas na solução deste problema, de forma que podemos conjecturar que:

Conjectura 4.1 O problema de computar numericamente, dada uma precisão $\varepsilon$, o valor esperado de uma função intervalar que denotamos aqui por $\left\langle f\left(x_{1}, \ldots, x_{n}\right)\right\rangle$ (equivalente a calcular uma integral multidimensional) com coeficientes racionais, $n$ variáveis aleatórias correlacionadas e $n$ entradas intervalares $I_{k}=\left[\underline{x}_{k}, \bar{x}_{k}\right]$ é NPDifícil.

Observação: Computar integrais multidimensionais pelos métodos numéricos como Regra de Simpson, do Trapézio, Integração Romberg e Quadraturas Gaussianas [8], requer tempos de computação $O\left(n_{\max }^{d}\right)$, onde $n_{\max }$ é o número de subdivisões dentre todos os intervalos $I_{k}, k=1, \ldots, n$ e $d$ é a dimensão do cálculo de integração.

Por outro lado, considerando que as variáveis aleatórias sejam independentes, isto é, $P\left(x_{1}, \ldots, x_{n}\right)=\prod_{i=1}^{n} P\left(x_{i}\right)$, pode-se calcular separadamente o valor esperado para uma função polinomial de várias variáveis, isto é, para cada variável particular, o que torna o problema pertencente a classe P.

Teorema 4.1. O problema de computar o valor esperado numericamente, dada uma precisão $\varepsilon$, de uma função racional $<f\left(x_{1}, \ldots, x_{n}\right)>$ com coeficientes racionais, $n$ variáveis aleatórias independentes e $n$ entradas intervalares $I_{k}=\left[\underline{x}_{k}, \bar{x}_{k}\right]$ é pertencente à classe $P$.

Demonstração. Considere uma função quadrática $f\left(x_{1}, \ldots, x_{n}\right)=\sum_{k=1}^{n} x_{k}^{2}+\sum_{k=1}^{n} x_{k}$. Para computar o valor esperado desta função, tem-se a seguinte equação:

$$
\left\langle f\left(x_{1}, \ldots, x_{n}\right)\right\rangle=\int_{x_{1} \in I_{1}} \ldots \int_{x_{n} \in I_{n}}\left(\sum_{k=1}^{n} x_{k}^{2}+\sum_{k=1}^{n} x_{k}\right) \prod_{i=1}^{n} P\left(x_{i}\right) d x_{i}
$$

a qual pode ser reduzida para:

$$
\begin{aligned}
& \left\langle f\left(x_{1}, \ldots, x_{n}\right)\right\rangle=\sum_{k=1}^{n}\left(\int_{x_{1} \in I_{1}} \ldots \int_{x_{n} \in I_{n}} x_{k}^{2} \prod_{i=1}^{n} P\left(x_{i}\right) d x_{i}\right)+ \\
& \sum_{k=1}^{n}\left(\int_{x_{1} \in I_{1}} \ldots \int_{x_{n} \in I_{n}} x_{k} \prod_{i=1}^{n} P\left(x_{i}\right) d x_{i}\right) .
\end{aligned}
$$

Sabe-se que $\int_{x \in I} P(x) d x=1$, conseqüentemente, a equação utilizada para computar o valor esperado da função $f\left(x_{1}, \ldots, x_{n}\right)$ com variáveis aleatórias independentes $x_{1}, \ldots, x_{n}$ é: 


$$
\left\langle f\left(x_{1}, \ldots, x_{n}\right)\right\rangle=\sum_{k=1}^{n}\left[\int_{I_{k}} x_{k}^{2} P\left(x_{k}\right) d x_{k}+\int_{I_{k}} x_{k} P\left(x_{k}\right) d x_{k}\right] .
$$

Observa-se que computar a integral definida $\int_{I_{k}} x_{k}^{2} P\left(x_{k}\right) d x_{k}$, significa calcular o valor esperado de uma única variável aleatória $x_{k}$ em um determinado intervalo $I_{k}$.

Considerando que o cálculo numérico de $\langle\bullet\rangle=\int_{I_{k}} x_{k} P\left(x_{k}\right) d x_{k}$ envolva $N_{k}$. $\operatorname{cop}_{\text {fmult }}$ (onde $N_{k}$ é o número de subdivisões de cada intervalo necessário para obter-se uma precisão $\varepsilon$, na estimativa do valor esperado e $c p_{f m u l t}$ indica a complexidade da operação fundamental de multiplicação), tem-se que na computação de $\left\langle f\left(x_{1}, \ldots, x_{n}\right)\right\rangle$, o número necessário de operações é

$$
C_{<f\left(x_{1}, \ldots, x_{n}\right)>}^{p}(n)=n \cdot \max _{k=1 \ldots n}\left\{N_{k}\right\} \cdot\left[\operatorname{cop}_{\text {fmult }}+c^{2} \text { op }_{\text {fmult }}\right],
$$

onde $C_{\left.<f\left(x_{1}, \ldots, x_{n}\right)\right\rangle}^{p}(n)$ indica a complexidade pessimista do cálculo do valor médio da função $f\left(x_{1}, \ldots, x_{n}\right)$.

Em outras palavras, o problema de computar o valor esperado de uma função $\left\langle f\left(x_{1}, \ldots, x_{n}\right)\right\rangle$ para variáveis aleatórias independentes é classificado como sendo um problema pertencente a classe $\mathrm{P}$, ou seja, o problema possui tempo polinomial para realizar tal computação deterministicamente. E desta forma o teorema está provado.

Deve-se ressaltar que, no presente trabalho, considera-se variáveis reais $x_{i}$ e, como entrada do problema de estimar o valor esperado, valores reais $x_{1}, \ldots, x_{n}$ onde $x_{1} \in I_{1}, \ldots, x_{n} \in I_{n}$. Dessa forma, o processamento de dados dá-se através de operações elementares de aritmética real.

\section{Amostragem por Importância como Heurística}

Com o propósito resolver e melhorar a complexidade do problema de computar numericamente, dada uma precisão $\varepsilon$, o valor esperado de uma função $f\left(x_{1}, \ldots, x_{n}\right)$, isto é, encontrar em tempo razoável uma solução mesmo que aproximada para a obtenção de valores esperados de funções de várias variáveis, aplica-se heurísticas com ênfase em algoritmos baseados no Método Monte Carlo.

O método Monte Carlo tem como base a utilização de variáveis pseudo-aleatórias para a resolução de um conjunto alargado de problemas. Sua aplicação constitui uma alternativa viável e flexível, não dependendo da dimensão da integração [7]. A aplicação do método é equivalente à resolução de um integral a $n$ dimensões, com condições fronteira que podem ser tão complexas quanto se queira.

Calcula-se a estimativa numérica do valor esperado de uma função $f\left(x_{1}, \ldots, x_{n}\right)$ de $n$ variáveis reais e $n$ valores amostrais $x_{k}$ (onde $\left(x_{1}, \ldots, x_{n}\right) \in \bigotimes_{k=1}^{n} I_{k}$ são gerados aleatóriamente), isto é, resolve-se a integral da equação (4.1) pelo método Monte Carlo através da seguinte equação:

$$
\left\langle f\left(x_{1}, \ldots, x_{n}\right)\right\rangle=\frac{1}{N_{\text {sample }}} \sum_{i=1}^{N_{\text {sample }}} f\left(x_{1}^{i}, \ldots, x_{n}^{i}\right) .
$$


Estima-se numericamente, dada uma precisão $\varepsilon$, o valor esperado de uma função racional com heurística (método Monte Carlo) trocando valores médios, mais complicados, por médias aritméticas simples, em um processo conhecido como Amostragem por Importância. O processo de amostragem por importância segue os seguintes passos:

- Selecionam-se as amostras $A_{i}=\left(x_{1}^{i}, \ldots, x_{n}^{i}\right) \in \bigotimes_{k=1}^{n} I_{k}, i=1, . ., N_{\text {sample }}$ $\left(N_{\text {sample }}=\right.$ número total de amostras $)$. Tais amostras são selecionadas de acordo com algum critério probabilístico que depende do contexto de cada problema;

- Estima-se o valor médio da função $f\left(x_{1}, \ldots, x_{n}\right)$ com os valores das amostras $A_{i}$ de acordo com a equação (5.1).

Este tipo de procedimento proporciona uma significativa redução do custo computacional em simulações onde requer-se um grande número de configurações.

Convém ressaltar que a principal desvantagem dessa heurística, isto é, do método Monte Carlo (com amostragem por importância), é que sua exatidão aumenta somente com $\sqrt{N_{\text {sample }}}$, ou seja, com o número de amostras [8]. Se a exatidão requerida é "modesta", ou se a capacidade de armazenamento do computador é grande, então pode-se recomendar o método como uma boa heurística para resolução de problemas de amostragem intervalar NP-Difíceis. Na próxima seção apresentaremos um exemplo de como essa Heurística pode ser utilizada para resolver o problema do cálculo do valor esperado para uma função de $n$ variáveis.

\subsection{Exemplo: Média configuracional em grafos regulares simples de grau 2}

Nesta subseção nos deteremos a mostrar um exemplo onde o método da amostragem por importância pode ser utilizado como uma Heurística apropriada para reduzir o tempo de computação de um problema com tempo notóriamente exponencial, que é o cálculo de médias configuracionais em um grafo regular simples de grau 2. Os vértices deste grafo regular podem assumir somente dois valores $-1 \mathrm{e}+1$, o que restringe os intervalos a serem todos discretos $I_{k}=[-1,1] \cap \mathbb{Z}^{*}, k=1, \ldots, n$. O referido exemplo apresenta heurísticas para o problema genérico de estimar numericamente, dada uma precisão $\varepsilon$, o valor esperado de uma função racional $f\left(x_{1}, \ldots, x_{n}\right)$ com $\left(x_{1}, \ldots, x_{n}\right) \in \prod_{k=1}^{n} \times I_{k}$ escolhidos de acordo com uma função de densidade de probabilidade $P\left(x_{1}, \ldots, x_{n}\right)$ conjunta, com $I_{k}$ sendo intervalos. Motivados pela Física Estatística (para uma básica introdução vide, por exemplo, [12]), vamos considerar um modelo muito simples para representar o que seria um material magnético unidimensional, denominado modelo de Ising [12]. Este problema pode ser olhado mais genéricamente como um grafo regular de grau 2 , com vértices $v_{1}, v_{2}, \ldots, v_{n}$, dispostos ordenadamente como na Figura 1 de forma que $v_{i}$ só é adjacente a $v_{i+1}$ e $v_{i-1}$, onde $i=1, \ldots, n$ e $v_{0}=v_{n}$ e $v_{n+1}=v_{1}$.

Vamos assumir que os vértices estejam associados, respectivamente, às variáveis $\sigma_{1}, \sigma_{2}, \ldots, \sigma_{n}$, onde $\sigma_{i}$, assume $q$ possíveis valores no conjunto $\Omega=\left\{d_{1}, d_{2}, \ldots, d_{q}\right\}$. No contexto do modelo de Ising este conjunto simplesmente se restringe a $\Omega=\{-1,+1\}$ 


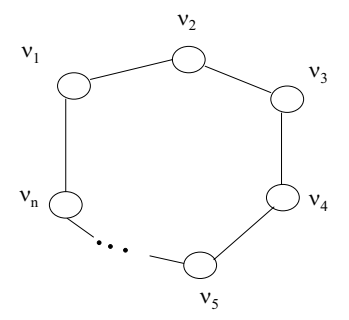

Figura 1: Grafo regular de grau 2, representando um magneto unifimensional. Cada vértice representa um íon magnético.

onde cada estado representa a projeção do "spin" (momento angular intrínseco) de um particular íon magnético, aqui representado por um vértice do grafo 1. Assumindo, então, que o custo entre dois vértices adjacentes $v_{i}$ e $v_{i+1}$ se dá simplesmente pelo produto $e\left(v_{1}, v_{2}\right)=-\sigma_{i} \sigma_{j}$, o custo em todo o grafo para uma particular configuração de spins $\left(\sigma_{1}, \sigma_{2}, \ldots, \sigma_{n}\right)$ seria dado simplesmente pela superposição:

$$
E\left(\sigma_{1}, \sigma_{2}, \ldots, \sigma_{n}\right)=-\sum_{i=1}^{n} \sigma_{i} \sigma_{i+1} .
$$

A função densidade de probabilidade $P(E)$ para um sistema deste tipo fora postulada por Ludwig Boltzmann (1844-1906) ser da forma exponencial:

$$
P\left[E\left(\sigma_{1}, \sigma_{2}, \ldots, \sigma_{n}\right)\right]=\frac{\exp \left[-\beta E\left(\sigma_{1}, \sigma_{2}, \ldots, \sigma_{n}\right)\right]}{\sum_{\substack{\sigma_{i}^{\prime} \in \Omega \\ i=1, \ldots, n}} \exp \left[-\beta E\left(\sigma_{1}^{\prime}, \sigma_{2}^{\prime}, \ldots, \sigma_{n}^{\prime}\right)\right]},
$$

onde $\beta$ é um parâmetro de expansão e no modelo representa o inverso da temperatura.

Calcular o valor esperado do custo no grafo acima definido significa calcular o valor esperado em relação a função densidade de probabilidade (5.2):

$$
\langle E\rangle=\frac{\sum_{\substack{\sigma_{i} \in \Omega \\ i=1, \ldots, n}} E\left(\sigma_{1}, \sigma_{2}, \ldots, \sigma_{n}\right) \exp \left[-\beta E\left(\sigma_{1}, \sigma_{2}, \ldots, \sigma_{n}\right)\right]}{\sum_{\substack{\sigma_{i} \in \Omega \\ i=1, \ldots, n}} \exp \left[-\beta E\left(\sigma_{1}, \sigma_{2}, \ldots, \sigma_{n}\right)\right]} .
$$

O cálculo dos somatórios nestas expressões envolvem notóriamente $O\left(2^{n}\right)$ operações. A amostragem por importância nos diz que escolhendo algumas configurações convenientemente através do algoritmo de Metrópolis [5], que privilegia baixas energias mas que ao mesmo tempo considera os efeitos de temperatura para a escolha das configurações apropriadas. Para aplicar essa Heurística, basicamente partimos de uma configuração inicial $\left(\sigma_{1}^{0}, \sigma_{2}^{0}, \ldots, \sigma_{n}^{0}\right)$ com custo $E_{0}$ e a partir disso realizamos uma pertubação (aleatória a princípio, isto é sorteia-se com probabilidade $1 / n$ se 


\begin{tabular}{ccccc}
\hline \hline$n$ & $\langle E\rangle_{\text {aprox }}^{N_{\text {sample }}=100}$ & $\langle E\rangle_{\text {aprox }}^{N_{\text {sample }}=1000}$ & $\langle E\rangle_{\text {aprox }}^{N_{\text {sample }}=10000}$ & $\langle E\rangle_{\text {exato }}$ \\
\hline \hline 10 & -7.52 & -8.22 & -7.87 & $-7.955661090 \ldots$ \\
20 & -14.04 & -15.18 & -15.33 & $-15.27921279 \ldots$ \\
30 & -21.48 & -23.05 & -22.76 & $-22.85250425 \ldots$ \\
& & & & \\
40 & -31.36 & -29.91 & -30.33 & $-30.46417598 \ldots$ \\
\hline \hline
\end{tabular}

Tabela 1: A primeira coluna representa o número de vértices utilizado para cada grafo pesquisado. Subseqüentemente a segunda, terceira e quarta colunas representa os valores esperados do custo obtidos pela Heurística para respectivamente 100, 1000, 10000 amostras para cada um dos valores de $n$ da primeira coluna. Na última coluna apresenta-se o valor exato obtido o valor esperado do custo.

a variável relacionada ao vértice é ou não alterada) nesta configuração altera-se em média apenas a variável de um vértice proporcionando uma nova e diferente configuração denotada aqui por $\left(\sigma_{1}^{1}, \sigma_{2}^{1}, \ldots, \sigma_{n}^{1}\right)$ com custo $E_{1}$.

Executando o procedimento e economizando bastante nos detalhes ${ }^{4}$, após a repetição do procedimento para um número $N_{\text {sample }}$ de amostras, a Heurística deve aproximar o valor 5.3, exponencialmente custoso $O\left(2^{n}\right)$, pela simples média aritmética $O\left(N_{\text {sample }}\right):\langle E\rangle_{\text {aprox }}=\frac{1}{N_{\text {sample }}} \sum_{i=1}^{\text {Nsample }} E_{i}$.

Ainda pode-se salientar que um limite de erro não rigoroso, para esta estimativa, pode ser calculada através do cálculo do desvio padrão do custo obtido nestas $N_{\text {sample }}$ amostras:

$$
\operatorname{error}(E)=\sqrt{\operatorname{var}(E)}=\sqrt{\frac{1}{N_{\text {sample }}} \sum_{i=1}^{N \text { sample }} E_{i}^{2}-\frac{1}{N_{\text {sample }}^{2}}\left(\sum_{i=1}^{N \text { sample }} E_{i}\right)^{2}} .
$$

Realizamos experimentos para $n=10,20,30$ e 40 onde comparamos os custo médio exato $\left(\langle E\rangle_{\text {exato }}\right)$ e o custo médio aproximado $\left(\langle E\rangle_{\text {aprox }}\right)$. O número de amostras $N_{\text {sample }}$ foi alterado para um estudo da robustez da heurística. Os resultados são apresentados na Tabela 1. Para esses resultados utilizamos $\beta=1.0$.

Como pode ser observado há uma excelente concordância visual entre os resultados obtidos heuristicamente com o valor exato. O aumento no número utilizado de amostras aumenta essa concordância.

\section{Conclusão}

O problema de estimar numericamente, dada uma precisão $\varepsilon$, o valor esperado de uma função racional $f\left(x_{1}, \ldots, x_{n}\right) \operatorname{com}\left(x_{1}, \ldots, x_{n}\right) \in \prod_{k=1}^{n} \times I_{k}$ escolhidos de acordo

\footnotetext{
${ }^{4}$ As implementações relativas e este artigo podem ser obtidas por e-mail diretamente com o autor Roberto da Silva (rdasilva@inf.ufrgs.br).
} 
com uma função de densidade de probabilidade $P\left(x_{1}, \ldots, x_{n}\right)$ conjunta, ou equivalentemente, o problema de calcular médias de funções de várias variáveis com entradas intervalares é NP-Difícil se considerarmos que as variáveis são correlacionadas. Porém, uma simplificação do problema ocorre se as variáveis aleatórias forem independentes, isto é, $P\left(x_{1}, \ldots, x_{n}\right)=P\left(x_{1}\right) \cdot \ldots \cdot P\left(x_{n}\right)$, e neste caso tem-se um problema pertencente a classe $\mathrm{P}$, ou seja, um problema que é solucionável em tempo polinomial por um algoritmo determinístico. Porém a maioria absoluta dos problemas envolvem variáveis correlacionadas, o que mostra que soluções alternativas para estes problemas são exigidas mesmo que não exatas.

Observou-se que este tipo de procedimento proporcionou uma significativa redução no custo computacional do problema que envolve o cálculo do valor esperado do custo energético de uma linha de ions magnéticos com condições de contorno periódicas e com interação somente entre vizinhos mais próximos (modelo de Ising), um exemplo que notóriamente requer o cálculo de custosas médias configuracionais. Os resultados mostraram uma razoável concordância do valor exato com a Heurística.

O método Monte Carlo possui uma desvantagem no que se refere a exatidão do valor numérico das médias aritméticas, ou seja, sua exatidão aumenta conforme o número de amostras $\sqrt{N_{\text {sample }}}$ necessárias para a solução de um determinado problema. Se a exatidão requerida na solução é "modesta", ou se a capacidade de armazenamento do computador é grande, recomenda-se a utilização do método em problemas que envolvam integrais multidimensionais.

Apesar desta "desvantagem", pode-se afirmar que o método constitui uma boa heurística para resolução de problemas de amostragem intervalar NP-Difíceis, pois verificou-se que o problema de estimar numericamente, dada uma precisão $\varepsilon$, uma função racional intervalar aplicado a esta heurística, sobre as $n$ variáveis aleatórias passou a pertencer a classe $\mathrm{P}$, isto é, o problema passou a ser solucionável em tempo polinomial através de um tratamento heurístico.

\begin{abstract}
The main objective of this work is to show alternatives for approximation of NP-Hard interval computation problem, using a heuristical approach with emphasis in algorithms based in the Monte Carlo Method. In order to present heuristics for the general problem of numerical estimating of the expected value of a rational function $f\left(x_{1}, \ldots, x_{n}\right)$ with $\left(x_{1}, \ldots, x_{n}\right) \in \prod_{k=1}^{n} \times I_{k} \subset \mathbb{R}^{n}$, given a precision $\varepsilon$, chosen in according to a joint probability density function $P\left(x_{1}, \ldots, x_{n}\right)$, where $I_{k}$ are intervals, we have showed, for the specific case of a regular graph of degree equal 2 , which nodes can assume the values -1 and +1 restricting all intervals be discrete $I_{k}=[-1,1] \cap \mathbb{Z}^{*}, k=1, \ldots, n$. We verified that expected value problem of between all adjacents nodes pair, had its complexity reduced from $O\left(2^{n}\right)$, using exact computing, to $O\left(N_{\text {sample }}\right)$ when approximated by Heuristic, where $N_{\text {sample }}$ is the number of sample of configurations chosen by the sampling importance process on the context of Methopolis algorithm.
\end{abstract}

\title{
Referências
}

[1] R.E. Campello e N. Maculan, "Algoritmos e Heurísticas: desenvolvimento e avaliação de performance", EDUFF, Rio de Janeiro, 1994. 
[2] M.E. Garey e D.S. Johnson, "Computers and intractability: a guide to the theory of NP-completeness", Freeman, San Francisco, 1979.

[3] V. Kreinovich, A. Lakeyev, J. Rohn e P. Kahl, "Computational Complexity and Feasibility of Data Processing and Interval Computations", Kluwer Academic Publishers, Dordrecht, 1998.

[4] V. Kreinovich, Probabilities, intervals, what next? Optimization problems related to extension of interval computations to situations with partial information about probabilities, Global Optimization, 29, No. 3 (2003), 265-280.

[5] N. Metropolis, W. A. Rosenbluth, M. N. Rosenbluth, A. H. Teller e E. Teller, Equation of state calculation by fast computer machines, J. Chem. Phys. 1, No. 6 (1953).

[6] R.E. Moore, "Methods and Applications of Interval Analysis", Society for Industrial and Applied Mathematics, Philadelphia, PA, 1979.

[7] R.M. Neal, "Probabilistic Inference using Markov Chain Monte Carlo Methods", Thecnical Report, Departament of Computer Science, University of Toronto, 1993.

[8] W.H. Press, S.A. Tenkolsky, W.T. Vetterling e B.P. Flannery, "Numerical Recipes in Fortran 77", 2 ed., Cambridge University Press, 1994.

[9] H. Rathschek e J. Rokne, "New Computer Methods for Global Optimization", Ellis Horwood Limited, Great Britain, 1988.

[10] L.V. Toscani e P.A.S. Veloso, "Complexidade de Algoritmos: análise, projetos e métodos", Sagra Luzzato, Instituto de Informática da UFRGS, Porto Alegre, 2001.

[11] B. Traylor e V. Kreinovich, A bright side of NP-hardness of interval computations: interval heuristics applied to NP-problems, Reliable Computing, 3 (1995), 343-359.

[12] S.R.A. Salinas, "Introdução a Física Estatística", Edusp, 1997. 